\title{
Is preoperative glenoid bone mineral density associated with aseptic glenoid implant loosening in anatomic total shoulder arthroplasty?
}

Sandrine Mariaux ${ }^{1}$, Raphaël Obrist ${ }^{2}$, Alain Farron ${ }^{1}$, Fabio Becce $^{3+}$ and Alexandre Terrier $^{1,2^{*+}}$ (ID

\begin{abstract}
Background: Aseptic loosening of glenoid implants is the primary revision cause in anatomic total shoulder arthroplasty (aTSA). While supported by biomechanical studies, the impact of glenoid bone quality, more specifically bone mineral density (BMD), on aseptic glenoid loosening remains unclear. We hypothesized that lower preoperative glenoid BMD was associated with aseptic glenoid implant loosening in aTSA.

Methods: We retrospectively included 93 patients (69 females and 24 males; mean age, 69.2 years) who underwent preoperative non-arthrographic shoulder computed tomography (CT) scans and aTSA between 2002 and 2014. Preoperative glenoid BMD (CT numbers in Hounsfield unit) was measured in 3D using a reliable semi-automated quantitative method, in the following six contiguous volumes of interest (VOI): cortical, subchondral cortical plate (SC), subchondral trabecular, and three successive adjacent layers of trabecular bone. Univariate Cox regression was used to estimate the impact of preoperative glenoid BMD on aseptic glenoid implant loosening. We further compared 26 aseptic glenoid loosening patients with 56 matched control patients.

Results: Glenoid implant survival rates were 89\% (95\% confidence interval Cl, 81-96\%) and 57\% (41-74\%) at 5 and 10 years, respectively. Hazard ratios for the different glenoid VOls ranged between 0.998 and $1.004(95 \% \mathrm{Cl}[0.996$, $1.007], p \geq 0.121)$. Only the SC VOI showed significantly lower CTn in the loosening group (622 $\pm 104 \mathrm{HU})$ compared with the control group $(658 \pm 88 \mathrm{HU})(p=0.048)$, though with a medium effect size $(\mathrm{d}=0.42)$. There were no significant differences in preoperative glenoid BMD in any other VOI between patients from the loosening and control groups.

(Continued on next page)
\end{abstract}

\footnotetext{
*Correspondence: alexandre.terrier@epfl.ch

${ }^{\dagger}$ Fabio Becce and Alexandre Terrier contributed equally to this work.

'Service of Orthopedics and Traumatology, Lausanne University Hospital and University of Lausanne, Avenue Pierre-Decker 4, 1011 Lausanne, Switzerland ${ }^{2}$ Laboratory of Biomechanical Orthopedics, Ecole Polytechnique Fédérale de Lausanne, Station 9, 1015 Lausanne, Switzerland

Full list of author information is available at the end of the article
}

C C The Author(s). 2021 Open Access This article is licensed under a Creative Commons Attribution 4.0 International License, which permits use, sharing, adaptation, distribution and reproduction in any medium or format, as long as you give appropriate credit to the original author(s) and the source, provide a link to the Creative Commons licence, and indicate if changes were made. The images or other third party material in this article are included in the article's Creative Commons licence, unless indicated otherwise in a credit line to the material. If material is not included in the article's Creative Commons licence and your intended use is not permitted by statutory regulation or exceeds the permitted use, you will need to obtain permission directly from the copyright holder. To view a copy of this licence, visit http://creativecommons.org/licenses/by/4.0/. The Creative Commons Public Domain Dedication waiver (http://creativecommons.org/publicdomain/zero/1.0/) applies to the data made available in this article, unless otherwise stated in a credit line to the data. 


\begin{abstract}
(Continued from previous page)
Conclusions: Although the preoperative glenoid BMD was statistically significantly lower in the SC region of patients with aseptic glenoid implant loosening compared with controls, this single-VOI difference was only moderate. We are thus unable to prove that lower preoperative glenoid BMD is clearly associated with aseptic glenoid implant loosening in aTSA. However, due to its proven biomechanical role in glenoid implant survival, we recommend extending this study to larger $C T$ datasets to further assess and better understand the impact of preoperative glenoid BMD on glenoid implant loosening/survival and aTSA outcome.
\end{abstract}

Keywords: Anatomic total shoulder arthroplasty, Aseptic loosening, Bone mineral density, Computed tomography, Glenoid implant

\section{Background}

The incidence of total shoulder arthroplasty (TSA) is increasing with population growth, aging, and the concomitant increase in prevalence of glenohumeral osteoarthritis (OA) [1-4]. Since the 1970s and the implantation of the first contemporary total shoulder prostheses, issues with radiolucent lines around glenoid components and aseptic loosening have been observed [5-7]. Despite major technological advances in prosthesis design, aseptic loosening of glenoid implants remains the primary cause of revision surgeries in anatomic TSA (aTSA) - affecting 0.01 to $6 \%$ of shoulder replacement surgeries - and its trend is increasing in proportion to the increase in TSA [5, 7-9].

The main risk factors for aseptic loosening of glenoid implants reported in the literature are related to biomechanical causes: malpositioning (in particular retroversion) of the glenoid component, head/glenoid implant diameter mismatch, persistence/recurrence of static posterior subluxation of the humeral head, and rotator cuff deficiency [10-14]. Indeed, all the above mentioned factors can cause impingements and/or excessive stress on the glenoid implant or at its bone interface. This may eventually lead to repeated micromotion and progressive implant loosening.

In addition to the aforementioned potential biomechanical causes of aseptic glenoid loosening in aTSA, glenoid bone quality has also been suggested and investigated as an additional contributor. Lower bone mineral density (BMD) has indeed been shown to increase the respective risks of periprosthetic fracture in total ankle arthroplasty [15] and pedicle screw loosening in spinal fusion surgery [16]. However, the precise role of preoperative glenoid BMD in aseptic glenoid loosening has not been thoroughly evaluated using a reliable quantitative CT method so far [17-22].

Terrier et al. assessed the association between CTderived preoperative glenoid BMD and several biomechanical predictions (cement stress, bone-cement interface stress, and bone strain) in aTSA, using patient-specific finite element models [22]. They showed that preoperative glenoid BMD was strongly negatively correlated with these biomechanical predictions, particularly in the subchondral trabecular bone, thus suggesting that this factor may contribute to aseptic loosening of glenoid implants [22]. However, to our knowledge, the clinical impact and relevance of the patient's preoperative glenoid BMD on glenoid implant survival in aTSA has not been evaluated in large case series yet.

Therefore, our objective was to test the hypothesis that aseptic loosening of glenoid implants in aTSA is associated with lower preoperative glenoid BMD. To do so, we retrospectively analyzed an institutional case series of aTSA patients, quantified the preoperative glenoid BMD in several predefined volumes of interest (VOI) from shoulder CT datasets, and evaluated its association with aseptic glenoid loosening.

\section{Methods}

\section{Patients and study design}

After approval by the institutional ethics committee (Lausanne University Hospital CER-VD, protocol 136/ $15)$, we retrospectively reviewed all consecutive cases treated with aTSA in our tertiary referral hospital between January 2002 and December $2014(n=262)$. All patients were operated through a deltopectoral approach by the same senior shoulder surgeon for the following clinical indications: primary glenohumeral OA $(n=200)$, post-traumatic glenohumeral OA $(n=31)$, avascular necrosis of the humeral head $(n=11)$, inflammatory arthritis $(n=12)$, or another diagnosis $(n=8)$. The cemented Aequalis all-polyethylene keeled glenoid component (Wright-Tornier, Bloomington, MN, USA) was implanted after minimum glenoid bone reaming to preserve the bone stock. Holes for keeled glenoid implants were drilled using proper instrumentation, and highviscosity bone cement was vacuum mixed and applied to fix them. All glenoid implants (small, medium, or large) were adapted to the size of the patient's glenoid cavity according to the manufacturer's recommendation chart for heads/glenoids diameter mismatch.

Of these 262 cases, we included all patients who had undergone preoperative shoulder CT scans $(n=184)$. The following exclusion criteria were subsequently applied: 
patients lost to follow-up ( $<2$ years) $(n=51)$, shoulder CT arthrography $(n=32)$, non-arthrographic shoulder CT scans with metal artifacts or incomplete CT coverage of the glenoid $(n=3)$, septic loosening of the glenoid implant $(n=3)$, glenoid implant malpositioning $(n=1)$, and recurrent postoperative scapulohumeral subluxation $(n=$ $1)$. CT arthrograms were excluded because the intraarticular iodinated contrast medium interfered with glenoid BMD measurements due to beam hardening artifacts [23, 24]. The resulting study population consisted of 93 patients, with a mean age at surgery of 69.2 years (range, 45.9-88.2 years), a female/male ratio of 69/24, a mean body mass index (BMI) of 27.4 (range, 18.4-42.4), and a smoking history in $10 / 93$ patients $(10.8 \%)$.

\section{Shoulder CT protocol}

All preoperative non-arthrographic shoulder CT scans were performed on 8-, 16-, or 64-detector row CT systems (LightSpeed Ultra, LightSpeed Pro 16, LightSpeed VCT, and Discovery CT750 HD; GE Healthcare, Waukesha, WI, USA) using the following standardized data acquisition settings: tube potential, 120-140 kVp; tube current, 144-440 mA; and gantry revolution time, $0.5-0.8 \mathrm{~s}$. The image reconstruction parameters were as follows: field of view $14 \times 14-32 \times 32 \mathrm{~cm}$ (thus yielding in-plane pixel sizes of $0.27 \times 0.27-0.63 \times 0.63 \mathrm{~mm}$ ); section thickness, $0.6-3.0 \mathrm{~mm}$; section interval, $0.3-2.0 \mathrm{~mm}$; and sharp (bone or bone plus, GE Healthcare) kernels.

\section{CT assessment of preoperative glenoid BMD and morphology}

Preoperative glenoid BMD was quantified in 3D from preoperative shoulder CT datasets using the same reliable method described in detail elsewhere [22]. Briefly, we measured the average CT numbers (CTn; in
Hounsfield unit, $\mathrm{HU}$ ) in six contiguous 3-mm-thick VOIs (cortical bone (CO), subchondral cortical plate (SC), subchondral trabecular bone (ST), and three successive adjacent layers of trabecular bone (T1, T2, and T3)) defined within a 40-mm-high cylinder aligned with the mediolateral scapular axis, centered on and adjusted to include the entire glenoid cavity, and whose medial base was positioned at the spinoglenoid notch (Fig. 1). Within this cylinder fully encompassing the glenoid, bone mineral tissue was then segmented using a lower threshold of $300 \mathrm{HU}$ [25]. All these measurements were performed using the Amira software (Thermo Fisher Scientific, Waltham, MA, USA) and user-defined Matlab scripts (MathWorks, Natick, MA, USA).

In addition, preoperative glenoid morphology was assessed on a picture archiving and communication system workstation (Vue PACS; Carestream Health, Rochester, NY, USA) by a board-certified orthopedic surgeon and a senior musculoskeletal radiologist independently, using the updated Walch grading system [26]. In the case of discrepancy, consensus was reached with a senior shoulder surgeon.

\section{Aseptic loosening of glenoid implants}

Aseptic loosening of glenoid implants was assessed using conventional shoulder radiographs (anteroposterior and axial/axillary, with/without lateral/scapular "Y" views) performed at regular follow-up visits at 3, 6, 12, and 24 (+/-1) months, followed by once a year or every 2 years, depending on the patient's clinical course. In the case of radiological uncertainty and/or onset of clinical symptoms (e.g. pain, feeling of instability or locking, decreased range of motion), shoulder CT arthrography was performed. Radiographic prosthetic loosening was defined as the presence and/or enlargement over time of

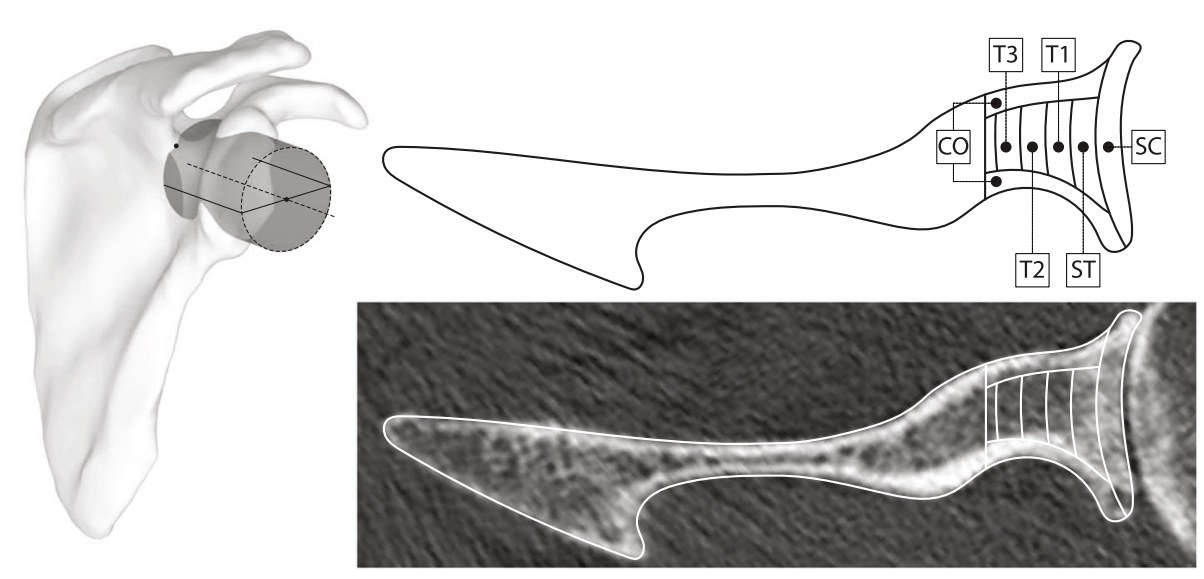

Fig. 1 Computed tomography numbers (in Hounsfield unit) were measured in six contiguous 3-mm-thick volumes of interest: cortical bone (CO), subchondral cortical plate (SC), subchondral trabecular bone (ST), and three successive adjacent layers of trabecular bone (T1, T2, and T3). All volumes of interest were defined within a 40-mm-high cylinder aligned with the mediolateral scapular axis, centered on and adjusted to include the entire glenoid cavity, and whose medial base was positioned at the spinoglenoid notch 
complete (thickness $>1.5 \mathrm{~mm}$ ) radiolucent lines at the glenoid bone-cement interface, and/or migration ( $>5$ $\mathrm{mm})$, tilt $\left(>5^{\circ}\right)$, or shift of the glenoid component $[2,5$, $8,27,28]$. The same observers as above independently reviewed all shoulder radiographs and, when available $(n=11)$, postoperative CT arthrograms, with the same consensus agreement approach in the case of discrepancy.

\section{Statistical analysis}

We first performed a Kaplan-Meier estimator to evaluate the survival (radiographic aseptic loosening as described above) of glenoid implants in the overall aTSA study population. Survival rate was assessed at 5 and 10 years postoperatively. This analysis was supplemented with univariate Cox proportional hazards regression models to estimate the effect of preoperative glenoid BMD (reflected by CTn in the various predefined VOIs) on glenoid implant survival (defined by the absence of aseptic glenoid loosening). We further analyzed the influence of gender, age, BMI, and smoking history on implant survival.

In a second step, we compared two patient groups. All patients with glenoid implant aseptic loosening were included in the loosening (LSG) group, while the control (CTR) group was formed by matching patients in terms of follow-up time, gender, age, BMI, smoking history, and Walch glenoid types. The follow-up time in the CTR group was at least 2 years. Continuous and categorical variables were compared using one-tailed independent-samples Student's t-test (or Wilcoxon signed-rank test in the case of skewed distribution with Lilliefors test) and chi-squared test, respectively. We tested the hypothesis that preoperative glenoid BMD (CTn) was numerically lower in the LSG than in the CTR group. Statistical significance was set at $p<0.05$. The effect size was measured using Cohen's d standardized difference between the two means, and interpreted as small $(\mathrm{d} \leq 0.2)$, medium $(\mathrm{d}=0.5)$, or large $(\mathrm{d} \geq 0.8)$ [29]. Statistical analysis was performed using Matlab's Statistics and Machine Learning Toolbox (MathWorks) by one of the authors (AT).

\section{Results}

The average follow-up time for the 93 patients included in our study was 6.2 years (range, 2.0-14.1 years). Among them, 26 (28.0\%) developed aseptic loosening of their glenoid implant according to our diagnostic criteria described above, which was confirmed in all cases intraoperatively. The Kaplan-Meier survival curve showed glenoid implant survival rates (absence of radiological loosening) of $89 \%$ (95\% confidence interval (CI), [81, $96 \%])$ and $57 \%(95 \% \mathrm{CI},[41,74 \%])$ at 5 and 10 years, respectively (Fig. 2). The hazard ratios (HR) of Cox

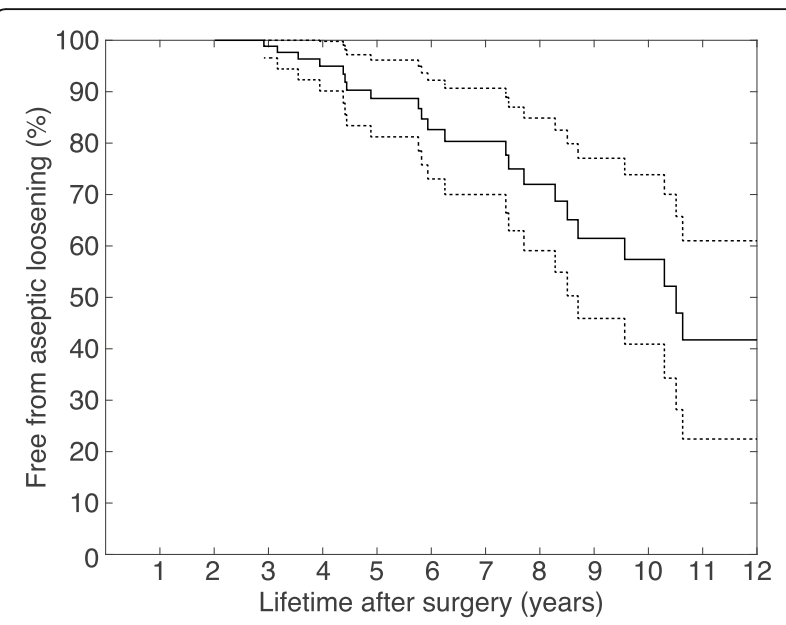

Fig. 2 Kaplan-Meier survival curve (solid line) for glenoid implants in patients with anatomic total shoulder arthroplasty, with the corresponding 95\% confidence intervals (dashed lines)

regressions for the different VOIs were very close to 1 (95\% CI, [0.996, 1.007]). Gender (HR, 0.900 (95\% CI, $[0.368,2.201]), p=0.817)$, age (HR, 1.001 (95\% CI, [0.952, 1.053]), $p=0.966)$, BMI (HR, 1.044 (95\% CI, [0.957, 1.139]), $p=0.332$ ), and smoking history (HR, 0.364, (95\% CI, $[0.049,2.717]), p=0.325)$ were also not significantly associated with aseptic glenoid loosening (Supplemental material).

The LSG group consisted of 26 patients: 19 females, 7 males; mean age, 67.5 years (range, 49.7-80.9 years); mean BMI, $26.8 \mathrm{~kg} / \mathrm{m}^{2}$ (range, $18.4-40.9 \mathrm{~kg} / \mathrm{m}^{2}$ ) (Table 1$)$. Walch glenoid types were as follows: A1 $(n=$ $4)$, A2 $(n=8)$, B1 $(n=4)$, and B2-B3 $(n=10)$. The mean follow-up time was 7.6 years (range, 2.9-14.1 years). On the other hand, the following 59 patients matched for follow-up time, gender, age, BMI, smoking history, and Walch glenoid types were included in the CTR group: 43 females, 16 males; mean age, 69.5 years (range, 45.988.2 years); mean BMI, $27.3 \mathrm{~kg} / \mathrm{m}^{2}$ (range, $19.1-41.2 \mathrm{~kg} /$ $\mathrm{m}^{2}$ ) (Table 1). Walch glenoid types were as follows: A1 $(n=7), \mathrm{A} 2(n=15), \mathrm{B} 1(n=17), \mathrm{B} 2-\mathrm{B} 3(n=16), \mathrm{C}(n=3)$, and $\mathrm{D}(n=1)$. The mean follow-up time in this CTR group was 6.1 years (range, $3.1-13.2$ years).

CTn were normally distributed in all different glenoid VOIs, except in ST of the LSG group. Only the SC VOI showed significantly lower CTn in the LSG group (622 $104 \mathrm{HU})$ compared with the CTR group $(658 \pm 88 \mathrm{HU})$ $(p=0.048)$, though with a medium effect size $(\mathrm{d}=0.420)$. There were no significant differences in CTn in any other VOI between patients from the LSG and CTR groups (Fig. 3 and Table 2).

\section{Discussion}

We hypothesized that lower preoperative glenoid BMD was associated with aseptic loosening of glenoid 
Table 1 Comparison of patient demographics and characteristics between the loosening (LSG) and control (CTR) groups

\begin{tabular}{llll}
\hline & LSG $(\boldsymbol{n}=\mathbf{2 6})$ & CTR $(\boldsymbol{n}=\mathbf{5 9})$ & $P$-value \\
\hline Gender & $19 \mathrm{~F} / 7 \mathrm{M}$ & $43 \mathrm{~F} / 16 \mathrm{M}$ & 0.985 \\
Age (years) & $67.5(49.7-80.9)$ & $69.5(45.9-88.2)$ & 0.362 \\
Body mass index $\left(\mathbf{k g} / \mathbf{m}^{2}\right)$ & $26.8(18.4-40.9)$ & $27.3(19.1-412)$ & 0.667 \\
Walch glenoid type & A1 $(n=4), \mathrm{A} 2(n=8), \mathrm{B} 1(n=4), \mathrm{B} 2-\mathrm{B} 3(n=10)$ & $\mathrm{A} 1(n=7), \mathrm{A} 2(n=15), \mathrm{B} 1(n=17), \mathrm{B} 2-3(n=16), \mathrm{C}(n=3), \mathrm{D}(n=1)$ & 0.644 \\
Follow-up time (years) & $7.6(2.9-14.1)$ & $6.1(3.1-13-2)$ & 0.055 \\
\hline
\end{tabular}

implants in aTSA. Although the preoperative glenoid BMD was statistically significantly lower in the SC region of patients with aseptic glenoid implant loosening compared with controls, this single-VOI difference between groups had only a moderate effect size. We were thus unable to prove that lower preoperative glenoid BMD is significantly associated with aseptic glenoid implant loosening in aTSA.

CT has previously been used to characterize the quality of the glenoid bone support and its relationship with aseptic glenoid implant loosening in aTSA. Chevalier et al. used micro finite element models based on microCT scans of cadaveric scapulae to evaluate the influence of bone volume fraction, trabecular anisotropy and cortical thickness on stress within the periprosthetic bone and cement mantle [19]. In a further computational study, Chen et al. measured the glenoid BMD in $\mathrm{HU}$ after simulated eccentric reaming for version correction of Walch B2 glenoids [18]. They analyzed BMD in five adjacent $1-\mathrm{mm}$ layers under the reamed glenoid surface, and concluded that increased version correction resulted in gradual depletion of high-quality bone from the anterior regions of B2 glenoids. Chamseddine et al. recently measured the glenoid BMD using a clinical quantitative CT technique on cadaveric scapulae where keeled or pegged cemented glenoid components were implanted

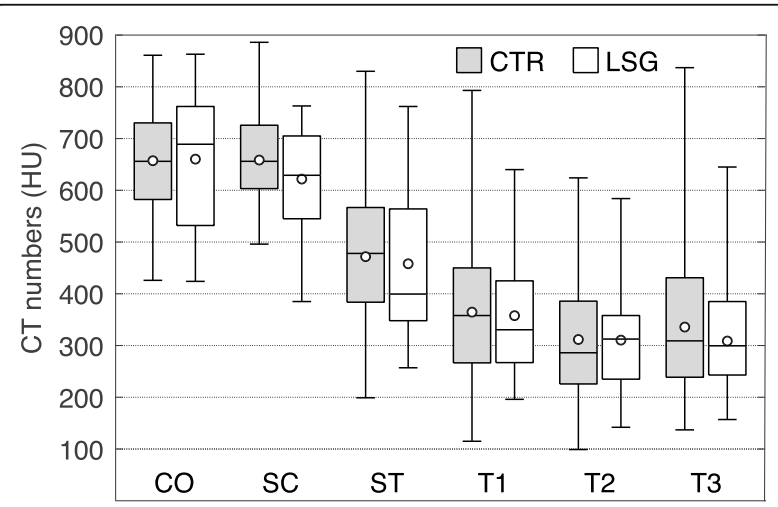

Fig. 3 Box-and-whisker plot showing CT numbers (in Hounsfield unit, $\mathrm{HU}$ ) for each of the six volumes of interest (cortical bone (CO), subchondral cortical plate (SC), subchondral trabecular bone (ST), and three successive adjacent layers of trabecular bone (T1, T2, and T3)) in patients with aseptic loosening of glenoid implants (LSG) compared with control (CTR) patients. Circles represent mean values
[17]. They divided the glenoid in different regions (inferior or superior, and inner, peripheral or full regions) and quantified BMD in $\mathrm{mg}$ of calcium hydroxyapatite per $\mathrm{cm}^{3}$. They reported that glenoids with lower BMD exhibited increased micromotion and displacement at the bone-implant interface, suggesting that implant failure most likely occur in glenoids with lower BMD, and that the fixation design may play a secondary role.

Other author groups have investigated and compared the glenoid BMD in various shoulder disorders. Couteau et al. initially compared the glenoid BMD in $\mathrm{HU}$ in patients with rotator cuff disorders, primary glenohumeral $\mathrm{OA}$, and rheumatoid arthritis using CT datasets and subdividing glenoids into $20 \mathrm{VOIs}$ [30]. They found that the glenoid BMD was higher centrally in patients with rotator cuff disorders, as opposed to primary OA where BMD was higher posteriorly and inferiorly. Divergent results were reported more recently by Harada et al. who reported, using CT osteoabsorptiometry in seven glenoid areas, a decrease in subchondral BMD in the central glenoid region of shoulders with symptomatic rotator cuff tears [31]. This variation in glenoid BMD was further emphasized by Knowles et al. who compared CTbased regional BMD and porosity in symmetric and asymmetric OA glenoid erosion patterns [32]. They subdivided glenoids in quadrants and two different $2.5-\mathrm{mm}$ depths. Concentric OA glenoids exhibited uniform subarticular BMD, while eccentric OA glenoids (Walch B2B3 types) showed densest bone with least porosity postero-inferiorly or in the neoglenoid region. Simon et al. reported similar results, while quantifying and characterizing in 3D the glenoid subchondral BMD with $\mathrm{CT}$ in five zones and three layers/depths [33]. They found that glenoid BMD varied depending on depth from the articular surface, topographic zone, and OA wear pattern. All these studies confirm the importance of evaluating glenoid BMD in several specific regions/ volumes rather than considering only one average density/CTn, when assessing its impact on aseptic loosening. However, none of these studies has yet investigated the association between preoperative glenoid BMD and aseptic loosening of glenoid implants in aTSA.

Our results confirm that the preoperative glenoid BMD gradually decreases with distance/depth from the articular surface. As expected and previously reported, 
Table 2 Average CT numbers (in Hounsfield unit) for each of the six volumes of interest (cortical bone (CO), subchondral cortical plate (SC), subchondral trabecular bone (ST), and three successive adjacent layers of trabecular bone (T1, T2, and T3)) in patients from the loosening (LSG) and control (CTR) groups, with the corresponding standard deviations (SD), minimum (Min) and maximum (Max) values, Cohen's d effect sizes, and p-values

\begin{tabular}{|c|c|c|c|c|c|c|c|c|c|c|c|c|}
\hline & \multicolumn{2}{|l|}{$\mathrm{CO}$} & \multicolumn{2}{|l|}{ SC } & \multicolumn{2}{|l|}{ ST } & \multicolumn{2}{|l|}{$\mathrm{T} 1$} & \multicolumn{2}{|l|}{ T2 } & \multicolumn{2}{|l|}{ T3 } \\
\hline & LSG & CTR & LSG & CTR & LSG & CTR & LSG & CTR & LSG & CTR & LSG & CTR \\
\hline Average & 660 & 657 & 622 & 658 & 458 & 472 & 358 & 365 & 310 & 312 & 309 & 338 \\
\hline SD & 124 & 104 & 104 & 88 & 138 & 135 & 115 & 139 & 105 & 118 & 105 & 137 \\
\hline Min & 424 & 426 & 385 & 496 & 257 & 199 & 196 & 115 & 142 & 99 & 157 & 137 \\
\hline Max & 853 & $861 c$ & 763 & 886 & 762 & 830 & 640 & 793 & 584 & 624 & 645 & 837 \\
\hline Cohen's d & 0.029 & & 0.420 & & 0.102 & & 0.049 & & 0.001 & & 0.197 & \\
\hline$P$-value & 0.547 & & 0.048 & & 0.224 & & 0.413 & & 0.483 & & 0.187 & \\
\hline
\end{tabular}

cortical bone regions were denser than trabecular bone regions [18, 32, 33]. We also observed that the SC BMD was lower in patients with aseptic glenoid implant loosening compared with CTR cases. It is unfortunately difficult to compare our results in terms of CTn (HU)/BMD with other studies because the measured glenoid regions/VOIs varied greatly among studies. Moreover, the vast majority of previous studies were biomechanically oriented, using computerized or cadaveric models, as opposed to ours which focused primarily on clinical outcome, more specifically aseptic loosening of glenoid implants. In our study, we first divided the glenoid into cortical and trabecular bone. Indeed, we avoided mixing cortical and trabecular bone, assuming that they could reasonably have different biomechanical properties and effects on the glenoid bone support. For the same reason, we subdivided cortical bone into eccentric and articular regions/VOIs, and trabecular bone into several contiguous layers at different depths from the articular surface. We then used $\mathrm{CTn}$ in $\mathrm{HU}$ as a surrogate for $\mathrm{BMD}$, as previously proven in the literature. Jun et al. recently analyzed with clinical CT imaging the architecture and mineralization of cadaveric glenoids compared to high-resolution micro-CT. They showed that clinical CT imaging was able to quantify regional (anatomic and peri-implant) variations in glenoid BMD [34]. Previously, Schreiber et al. demonstrated that CTn in HU correlated well with BMD and compressive strengths as measured with dual $x$-ray absorptiometry scans and mechanical testing of synthetic bone models [35]. Most recently, a CT study reported that OA glenoids had a higher BMD than normal glenoids, with higher $\mathrm{CTn}$ in the SC region particularly posteriorly, which is consistent with our findings [36].

Our study shows a $28 \%$ rate of aseptic glenoid implant loosening at 6.2 years and $57 \%$ survival rate at 10 years follow-up, which differ from the rates reported in the literature. In a review, Gonzalez et al. found a $14.3 \%$ rate of aseptic loosening in a series of 2657 aTSAs with a mean follow-up of 6 years [37]. However, Walch et al., in a multicenter study based on radiographic analysis [38], and Denard et al. both reported higher aseptic loosening rates of 32 and $43 \%$ at mean follow-ups of 8.5 and 9.5 years, respectively; the latter study in patients aged 55 years or younger [39]. On the other hand, the estimated survival rate of aTSA is $83-95 \%$ at 10 years $[5,40,41]$. However, the glenoid implant survival rate reported in our study is biased for several reasons. First, our study included B2-B3 glenoids and patients with inflammatory arthritis, which could increase complication rates. Indeed, the indications for TSA have changed and narrowed over time [42]. Second, we had to exclude a substantial number of patients because they did not underwent preoperative shoulder CT scans or only shoulder CT arthrograms which could not be used here. All this had an influence on our reported lower 10-year glenoid implant survival rate.

To our knowledge, our study is the first to quantitatively assess the impact of preoperative glenoid BMD in 3D on glenoid implant survival - more specifically aseptic glenoid loosening - in aTSA. Most previous studies have evaluated the quality of the glenoid bone support using CT datasets (either micro- or conventional CT), but only few have correlated their findings with clinical and radiological outcomes $[17-19,22]$. Our semiautomated quantitative measurement method based on a computerized 3D scapular reconstruction model has proven its reliability and already helped improving glenoid implant positioning [43]. This method further allows an in-depth analysis with subdivision of the glenoid bone and its region-specific BMD, distinguishing between the various cortical and trabecular regions. The technique is being made fully automated using deep learning, which should enable future rapid analysis of large clinical CT datasets.

Among the major limitations of our study are the inherent weaknesses of a retrospectively designed study and the relatively high number of patients lost to followup. Furthermore, the minimum follow-up was only 2 years, which may have underestimated cases with aseptic 
glenoid implant loosening occurring at mid- or longterm. However, the average follow-up time for LSG and CTR groups was longer and appropriate. Although aseptic glenoid implant loosening is one of the main complications in aTSA, the prosthetic survival rate is estimated to range between 95 and $99 \%$ and $83-95 \%$ at 5 and 10 years, respectively $[5,41,44]$. Despite recommendations for regular follow-up, patients with favorable clinical outcome tend not to show up at scheduled follow-up visits. Another study limitation is the lack of clear definition and consensus for the diagnosis of aseptic glenoid implant loosening. The most widely used definition from Cofield states that aseptic loosening is a shift and medialization of the glenoid component usually accompanied by superior tilting of the glenoid prosthetic surface on plain radiographs $[45,46]$. A more recent definition by Martin et al. defined aseptic loosening as migration of more than $5 \mathrm{~mm}$ or tilt of more than 5 degrees of the glenoid implant [28]. However, smaller displacements or enlargements over time of radiolucent lines at the bonecement interface may also be seen in patients with aseptic glenoid implant loosening [28]. Furthermore, the distinction between radiolucent lines around the glenoid implant, which are relatively common (10-94\%) and usually asymptomatic $[27,47,48]$, and aseptic loosening remains unclear. A further limitation is the absence of systematic postoperative shoulder CT scans to assess both the accurate position of the glenoid implant relative to the glenoid VOIs measured preoperatively, and the glenoid bone-cement interface postoperatively. Indeed, depending on intraoperative glenoid bone reaming (patients with B2-B3 glenoid types all underwent minimum anterior reaming in our series, $n=7$ ), the bone region on which the glenoid implant was lying may be slightly different/offset from the corresponding VOI measured on preoperative CT. Our results should also be weighed by missing retrospective data on and analysis of potential confounding factors for aseptic glenoid implant loosening, such as patients' comorbidities (e.g. diabetes) and activity level. Finally, the type of glenoid implant used may also have an influence on our results. During the study inclusion period, only keeled glenoid implants were implanted in our institution. Recent data show that such glenoid implant designs are associated with a slightly increased rate of aseptic loosening compared with pegged implants [49].

\section{Conclusions}

Although we observed lower preoperative glenoid BMD in the SC region of patients with aseptic glenoid implant loosening compared with matched CTR cases, this statistical difference was only moderate (medium effect size) and not found in any other glenoid regions. We were thus unable to assert that lower preoperative glenoid
BMD is clearly associated with aseptic loosening of glenoid implants in aTSA. However, due to the proven biomechanical role of the bone support in glenoid implant survival, we still recommend extending this initial study to larger CT datasets with higher follow-up rates to further assess and better define the real impact of the preoperative glenoid BMD on aTSA outcome overall and glenoid implant survival in particular. Finally, the methods and results presented here might eventually be implemented in preoperative planning softwares, and used to aid in better preparing the implantation of glenoid components in aTSA.

\section{Supplementary Information}

The online version contains supplementary material available at https://doi. org/10.1186/s12891-020-03892-0.

Additional file 1: Supplementary material. Univariate Cox

proportional hazards regression and Forest plot.

\section{Abbreviations}

aTSA: Anatomic total shoulder arthroplasty; BMD: Bone mineral density; BMI: Body mass index; CO: Cortical bone; CT: Computed tomography; CTn: Computed tomography number; CTR: Control; HR: Hazard ratio; HU: Hounsfield unit; LSG: Loosening; OA: Osteoarthritis; SC: Subchondral cortical plate; ST: Subchondral trabecular bone; TSA: Total shoulder arthroplasty; T1-T3: Layers 1-3 of trabecular bone; VOI: Volume of interest

\section{Acknowledgements}

Not applicable.

\section{Authors' contributions}

SM collected the patient clinical data, participated to data interpretation and manuscript writing. RO implemented the measurement method and performed the measurements. AF proposed the study, supervised its design, and participated to data interpretation and manuscript writing. FB provided the patient $C T$ data, participated to data interpretation and manuscript writing. AT supervised the methodology, performed the statistical analyses, and participated to data interpretation and manuscript writing. All authors read and approved the final manuscript.

Funding

This study was funded by the "Lausanne Orthopedic Research Foundation" and Tornier-Wright (Bloomington, MN, USA).

\section{Availability of data and materials}

The datasets used and/or analyzed during the current study are available from the corresponding author on reasonable request.

\section{Ethics approval and consent to participate}

This study was approved by the institutional ethics committee "Commission cantonale d'éthique de la recherche sur l'être humain (CER-VD)" (protocol

$136 / 15)$, with waiver of informed consent.

Consent for publication

Not applicable.

\section{Competing interests}

The authors declare that they have no competing interests.

\section{Author details}

'Service of Orthopedics and Traumatology, Lausanne University Hospital and University of Lausanne, Avenue Pierre-Decker 4, 1011 Lausanne, Switzerland. ${ }^{2}$ Laboratory of Biomechanical Orthopedics, Ecole Polytechnique Fédérale de Lausanne, Station 9, 1015 Lausanne, Switzerland. ${ }^{3}$ Department of Diagnostic 
and Interventional Radiology, Lausanne University Hospital and University of Lausanne, Rue du Bugnon 46, 1011 Lausanne, Switzerland.

\section{Received: 27 August 2020 Accepted: 16 December 2020 Published online: 08 January 2021}

\section{References}

1. Day JS, Lau E, Ong KL, Williams GR, Ramsey ML, Kurtz SM. Prevalence and projections of total shoulder and elbow arthroplasty in the United States to 2015. J Shoulder Elb Surg. 2010;19:1115-20.

2. Kasten P, Pape G, Raiss P, Bruckner T, Rickert M, Zeifang F, et al. Mid-term survivorship analysis of a shoulder replacement with a keeled glenoid and a modern cementing technique. J Bone Joint Surg Br. 2010:92:387-92.

3. Kim SH, Wise BL, Zhang Y, Szabo RM. Increasing incidence of shoulder arthroplasty in the United States. J Bone Joint Surg Am. 2011:93:2249-54.

4. Rasmussen JV, Hole R, Metlie T, Brorson S, Äärimaa V, Demir Y, et al. Anatomical total shoulder arthroplasty used for glenohumeral osteoarthritis has higher survival rates than hemiarthroplasty: a Nordic registry-based study. Osteoarthr Cartil. 2018:26:659-65.

5. Collin P, Tay AKL, Melis B, Boileau P, Walch G. A ten-year radiologic comparison of two-all polyethylene glenoid component designs: a prospective trial. J Shoulder Elb Surg. 2011;20:1217-23.

6. Mileti J, Boardman ND, Sperling JW, Cofield RH, Torchia ME, O'driscoll SW, et al. Radiographic analysis of polyethylene glenoid components using modern cementing techniques. J Shoulder Elb Surg. 2004;13:492-8.

7. Neer CS, Watson KC, Stanton FJ. Recent experience in total shoulder replacement. J Bone Joint Surg Am. 1982;64:319-37.

8. Bohsali Kl, Wirth MA, Rockwood CA. Complications of total shoulder arthroplasty. J Bone Joint Surg Am. 2006;88:2279-92.

9. Wirth MA, Rockwood CA. Complications of total shoulder-replacement arthroplasty. J Bone Joint Surg Am. 1996;78:603-16.

10. Ho JC, Sabesan VJ, lannotti JP. Glenoid component retroversion is associated with osteolysis. J Bone Joint Surg Am. 2013;95:e82.

11. lannotti JP, Norris TR. Influence of preoperative factors on outcome of shoulder arthroplasty for glenohumeral osteoarthritis. J Bone Joint Surg Am. 2003:85-A:251-8.

12. Matsen FA, Clinton J, Lynch J, Bertelsen A, Richardson ML. Glenoid component failure in total shoulder arthroplasty. J Bone Joint Surg Am. 2008;90:885-96.

13. Walch G, Edwards TB, Boulahia A, Boileau P, Mole D, Adeleine P. The influence of glenohumeral prosthetic mismatch on glenoid radiolucent lines: results of a multicenter study. J Bone Joint Surg Am. 2002;84-A: 2186-91.

14. Hasler A, Meyer DC, Tondelli T, Dietrich T, Gerber C. Radiographic performance depends on the radial glenohumeral mismatch in total shoulder arthroplasty. BMC Musculoskelet Disord. 2020;21:206.

15. Cody EA, Lachman JR, Gausden EB, Nunley JA, Easley ME. Lower bone density on preoperative computed tomography predicts periprosthetic fracture risk in total ankle arthroplasty. Foot Ankle Int. 2019;40:1-8.

16. Bredow J, Boese CK, Werner CML, Siewe J, Löhrer L, Zarghooni K, et al. Predictive validity of preoperative CT scans and the risk of pedicle screw loosening in spinal surgery. Arch Orthop Trauma Surg. 2016;136:1063-7.

17. Chamseddine M, Breden S, Pietschmann MF, Müller PE, Chevalier Y. Periprosthetic bone quality affects the fixation of anatomic glenoids in total shoulder arthroplasty: in vitro study. J Shoulder Elbow Surg. 2019;28(1):e1828. https://doi.org/10.1016/j.jse.2018.07.012.

18. Chen X, Reddy AS, Kontaxis A, Choi DS, Wright T, Dines DM, et al. Version correction via eccentric reaming compromises remaining bone quality in B2 glenoids: a computational study. Clin Orthop. 2017:475:3090-9.

19. Chevalier Y, Santos I, Müller PE, Pietschmann MF. Bone density and anisotropy affect periprosthetic cement and bone stresses after anatomical glenoid replacement: a micro finite element analysis. J Biomech. 2016;49: 1724-33.

20. Merolla G, Amore B, Paladini P, Cavagna E, Porcellini G. Computed tomography quantification of bone density adjacent to cemented pegged polyethylene glenoid components in shoulder arthroplasty. Eur J Orthop Surg Traumatol Orthop Traumatol. 2014;24:753-61.

21. Sowa B, Bochenek M, Braun S, Kretzer JP, Zeifang F, Bruckner T, et al. The subchondral bone layer and glenoid implant design are relevant for primary stability in glenoid arthroplasty. Arch Orthop Trauma Surg. 2018;138:1487-94
22. Terrier A, Obrist R, Becce F, Farron A. Cement stress predictions after anatomic total shoulder arthroplasty are correlated with preoperative glenoid bone quality. J Shoulder Elb Surg. 2017;26:1644-52.

23. He T, Qian X, Zhai R, Yang Z. Computed tomography number measurement consistency under different beam hardening conditions: comparison between dual-energy spectral computed tomography and conventional computed tomography imaging in phantom experiment. J Comput Assist Tomogr. 2015;39:981-5.

24. Meganck JA, Kozloff KM, Thornton MM, Broski SM, Goldstein SA. Beam hardening artifacts in micro-computed tomography scanning can be reduced by $X$-ray beam filtration and the resulting images can be used to accurately measure BMD. Bone. 2009;45:1104-16.

25. Fullerton GD, White DR. Anthropomorphic test objects for CT scanners. Radiology. 1979;133:217-22.

26. Bercik MJ, Kruse K, Yalizis M, Gauci M-O, Chaoui J, Walch G. A modification to the Walch classification of the glenoid in primary glenohumeral osteoarthritis using three-dimensional imaging. J Shoulder Elb Surg. 2016:25:1601-6.

27. Lazarus MD, Jensen KL, Southworth C, Matsen FA. The radiographic evaluation of keeled and pegged glenoid component insertion. J Bone Joint Surg Am. 2002;84-A:1174-82.

28. Martin SD, Zurakowski D, Thornhill TS. Uncemented glenoid component in total shoulder arthroplasty. Survivorship and outcomes. J Bone Joint Surg Am. 2005;87:1284-92.

29. Sullivan GM, Feinn R. Using effect size-or why the $p$ value is not enough. J Grad Med Educ. 2012;4:279-82.

30. Couteau B, Mansat P, Mansat M, Darmana R, Egan J. In vivo characterization of glenoid with use of computed tomography. J Shoulder Elb Surg. 2001;10: $116-22$

31. Harada Y, Yokoya S, Akiyama Y, Mochizuki Y, Ochi M, Adachi N. Bone mineralization changes of the glenoid in shoulders with symptomatic rotator cuff tear. Int Orthop. 2018;42:2639-44.

32. Knowles NK, Athwal GS, Keener JD, Ferreira LM. Regional bone density variations in osteoarthritic glenoids: a comparison of symmetric to asymmetric (type B2) erosion patterns. J Shoulder Elb Surg. 2015;24:425-32.

33. Simon P, Gupta A, Pappou I, Hussey MM, Santoni BG, Inoue N, et al. Glenoid subchondral bone density distribution in male total shoulder arthroplasty subjects with eccentric and concentric wear. J Shoulder Elb Surg. 2015;24: 416-24.

34. Jun B-J, Vasanji A, Ricchetti ET, Rodriguez E, Subhas N, Li Z-M, et al. Quantification of regional variations in glenoid trabecular bone architecture and mineralization using clinical computed tomography images. J Orthop Res Off Publ Orthop Res Soc. 2018;36:85-96.

35. Schreiber JJ, Anderson PA, Rosas HG, Buchholz AL, Au AG. Hounsfield units for assessing bone mineral density and strength: a tool for osteoporosis management. J Bone Joint Surg Am. 2011;93:1057-63.

36. Letissier H, Chaoui J, Bercik MJ, Boileau P, Le Nen D, Stindel E, et al. Glenoid subchondral bone density in osteoarthritis: a comparative study of asymmetric and symmetric erosion patterns. Orthop Traumatol Surg Res. 2020;106:1127-34.

37. Gonzalez J-F, Alami GB, Baque F, Walch G, Boileau P. Complications of unconstrained shoulder prostheses. J Shoulder Elb Surg. 2011;20:666-82.

38. Walch G, Young AA, Boileau P, Loew M, Gazielly D, Molé D. Patterns of loosening of polyethylene keeled glenoid components after shoulder arthroplasty for primary osteoarthritis: results of a multicenter study with more than five years of follow-up. J Bone Jt Surg - Ser A. 2012;94:145-50.

39. Denard PJ, Raiss P, Sowa B, Walch G. Mid- to long-term follow-up of total shoulder arthroplasty using a keeled glenoid in young adults with primary glenohumeral arthritis. J Shoulder Elb Surg. 2013;22:894-900.

40. Fevang B-TS, Lie SA, Havelin LI, Skredderstuen A, Furnes O. Risk factors for revision after shoulder arthroplasty. Acta Orthop. 2009;80:83-91.

41. McLendon PB, Schoch BS, Sperling JW, Sánchez-Sotelo J, Schleck CD, Cofield RH. Survival of the pegged glenoid component in shoulder arthroplasty: part II. J Shoulder Elb Surg. 2017;26:1469-76.

42. Walch G, Moraga C, Young A, Castellanos-Rosas J. Results of anatomic nonconstrained prosthesis in primary osteoarthritis with biconcave glenoid. J Shoulder Elb Surg. 2012;21:1526-33.

43. Terrier A, Ston J, Larrea X, Farron A. Measurements of three-dimensional glenoid erosion when planning the prosthetic replacement of osteoarthritic shoulders. Bone Jt J. 2014;96-B:513-8.

44. Fevang BTS, Nystad TW, Skredderstuen A, Furnes ON, Havelin LI. Improved survival for anatomic total shoulder prostheses. Acta Orthop. 2015;86:63-70. 
45. Cofield RH. Total shoulder arthroplasty with the Neer prosthesis. J Bone Joint Surg Am. 1984;66:899-906.

46. Nagels J, Valstar ER, Stokdijk M, Rozing PM. Patterns of loosening of the glenoid component. J Bone Joint Surg Br. 2002;84:83-7.

47. Barwood S, Setter KJ, Blaine TA, Bigliani LU. The incidence of early radiolucencies about a pegged glenoid component using cement pressurization. J Shoulder Elb Surg Am Shoulder Elb Surg Al. 2008;17:703-8.

48. Fox TJ, Foruria AM, Klika BJ, Sperling JW, Schleck CD, Cofield RH.

Radiographic survival in total shoulder arthroplasty. J Shoulder Elb Surg Am Shoulder Elb Surg Al. 2013;22:1221-7.

49. Dillon MT, Chan PH, Prentice HA, Burfeind WE, Yian EH, Singh A, et al. The association between glenoid component design and revision risk in anatomic total shoulder arthroplasty. J Shoulder Elb Surg. 2020. https://doi. org/10.1016/j.jse.2020.02.024.

\section{Publisher's Note}

Springer Nature remains neutral with regard to jurisdictional claims in published maps and institutional affiliations.

Ready to submit your research? Choose BMC and benefit from:

- fast, convenient online submission

- thorough peer review by experienced researchers in your field

- rapid publication on acceptance

- support for research data, including large and complex data types

- gold Open Access which fosters wider collaboration and increased citations

- maximum visibility for your research: over $100 \mathrm{M}$ website views per year

At BMC, research is always in progress.

Learn more biomedcentral.com/submissions 